\title{
On the problem of optimizing the door hinge of electro car by generative design methods
}

\author{
R. Petrov ${ }^{1}$, P. Cvetkov, A. Maksimov, O. Klyavin \\ Peter the Great St.Petersburg Polytechnic University (SPbPU) \\ Russia
}

Received: May 28, 2020. Revised: June 25, 2020. Accepted: July 1, 2020. Published: July 21, 2020.

\begin{abstract}
Our research describes the optimization of car door hinges. The model we are considering is a pair of upper and lower hinges of car doors and cases of their loading. Optimization of this part of the vehicle consists in reducing the mass of the product, which is taken as the target function, while maintaining the mechanical characteristics within acceptable values. The characteristics of various types of manufacturing, such as milling, casting, and additive manufacturing, were also used as optimization criteria. During the research the authors have undertaken the task of using the most advanced approaches for calculation, optimization and analysis of their results, which are the use of special calculation systems, calculations that are performed on the GPU, what is the way much reduces the required optimization time, methods of generative design to achieve the required criteria and consideration of possible methods of manufacture of the workpiece in terms of additive manufacturing. The result of our research is the concept of optimizing vehicle door hinges and selecting the appropriate production method. As a test of the positive effect of the applied method, a repeated static calculation of the structure was made, based on the optimized geometry.
\end{abstract}

Keywords-Door hinge, generative design, GPU, parallel computing.

\section{INTRODUCTION}

$\mathrm{T}$ HERE is currently no precise, well-established definition of "generative design". In various resources, you can find information about where generative design is called "an approach to design in which a person delegates part of the processes to computer technologies and platforms". You can also find similar statements: "Newest software based on

${ }^{1}$ Corresponding author: petrov.ri@edu.spbstu.ru

The work was performed with financial support of the Ministry of Science and Higher Education of the Russian Federation as part of the implementation of the Federal Target Program "Research and development in priority areas of the scientific and technological complex of Russia for 2014-2020". Unique identifier of the agreement RFMEFI57818X0269. artificial intelligence, which is able to generate structures and parts according to the specified parameters almost without the participation of engineers." These statements are

fundamentally incorrect, starting from the fact that engineers have been delegating part of the processes to software packages for more than a quarter of a century, including strength calculations and optimization, which does not exclude the engineer from the process in any way, because a certain set of skills and knowledge is needed for a competent task statement, even using engineering software. To the fact that the results will be "generated" by the computer the generated results obviously have to be checked by the compliance of the mechanical characteristics of the received detail and a process engineer who must assess the possibility and economic feasibility of manufacturing the part.

So, generative design differs from topological optimization in the presence of algorithms used based on the framework to redefine the area remaining after optimization calculation for the required tasks. For example, we know the parameters of the cutting tool that we will use to manufacture the product, as well as how many axes our milling machine can use. The program will consider the possible path of the cutter in order to interpret the model in such a way that it can be produced without much difficulty on the equipment we have.

Due to the circumstances described above, we will try to give the definition of generative design.

Generative design is an optimization process based on the use of various additional, auxiliary, algorithms that serve to generate the optimization domain (including algorithms that consider the manufacturing process). For example, the path along which the cutting tool of a milling machine goes) and has a functional that can, as a result, output a ready-made solid-state model. For the proposed approach to design, different terms are also used: there are "heavy", - for example, the "generative design" introduced by Autodesk, i.e. the structure is generated during design, and often in automatic mode, without the participation of the designer, due to algorithms. These algorithms are based on machine learning techniques for training artificial intelligence, the so-called "engineering intuition", due to which the neural network simultaneously serves as an assistant both in the construction of a mathematical model of the optimized product and in the 
process of its interpretation, taking into account, for example, manufacturing parameters.

This article contributes to the body of engineering knowledge, and specifically to the field of optimization in the mechanics of a deformable solid in which the authors turn to the use of new techniques in the approach to optimization.

The paper describes the approach to optimization described above and compares the results obtained on the same model in one of the most widely used Ansys optimizer and the generative design program.

\section{MATERIALS AND METHODS}

The most studied and developed mathematical model of optimization is SIMP optimization method, and this approach is used in most software for implementing topological optimization [1]. As in the homogenization approach, the volume of material to be optimized is divided into a grid of $N$ elements (isotropic solid microstructures). Each element is mapped to a relative density of $\rho_{\mathrm{e}}$. The target function is the strain energy. Restrictions are imposed on the volume of the material. Thus, the problem of topology optimization using the SIMP method can be formulated as follows:

$$
\begin{aligned}
& \text { Minimize: } S E\left(\rho_{e}\right)=\sum_{e=1}^{N}\left(\rho_{e}\right)^{p} u_{e}^{T} k_{e} u_{e} ; \\
& \text { Restrictions: } \frac{V(x)}{V_{0}}=f ; \\
& K U=F ; \\
& 0<\rho_{\min } \leq \rho_{e} \leq 1
\end{aligned}
$$

Where $U-$ the global displacement vector; $F$ - global vector of forces; $u_{e}$ and $k_{e}$ - displacement vector and the stiffness matrix of the element; $\rho_{e}-$ vector of design parameters (vector of the relative densities of the elements), and $\rho_{\text {min }}$-minimum density value (for empty elements), nonzero for stability of finite-element analysis; $N$ is the number of elements in the model; $V(x)$ - current volume of the structure; $V_{0}$ - initial volume of the structure; $f$ - given in advance the ratio of change of volume, that is, selection of the necessary amount of material for disposal; $p$ - penalty parameter; $x$ vector of constructive variables.

The objective function and constraints variables that can be obtained through finite element analysis. The choice of the vector of constructive variables $x$ depends on the type of optimization being performed. For topological optimization, the design variables are element densities. When optimizing the size, design variables are properties of structural elements. In topographic optimization, design variables are represented as a linear combination of a modified shape.

The value of the material density of each finite element is dimensionless and varies between 0 and 1, where 1 indicates the need to preserve this element, and 0 , conversely, means that it must be removed. Unfortunately, optimization over a large number of discrete variables cannot be implemented numerically. Consequently, the problem of material distribution is posed in continuous variables. The stiffness of the material is assumed to be linearly dependent on the thickness. To reduce the density of the material distribution to the interval $[0 ; 1]$, the formula is used:

$K^{p}=\rho^{p} K$,

$\rho$ - density of the element (the reduced thickness of the material), and $\mathrm{p}$ is the resulting coefficient, which is always greater than one.

In this mathematical method, additional restrictions may be imposed, for example, you can change the algorithm when optimizing a part that will later be obtained by casting. In this case, all elements on the line $\mathrm{k}$ parallel to the casting direction must be assigned increasing densities [2]:

$\left(0 \leq \rho_{1} \leq \rho_{2} \leq \ldots \leq 1\right)_{k=1 \ldots K}$

The results obtained using finite element analysis at the first step of each iteration are used to calculate the sensitivity of each element. Sensitivity is called the impulse, or the effect that a variation in the density of an element has on the target function. The expression for sensitivity can be written as follows:

$\frac{\delta S E}{\delta P}=-p\left(\rho_{e}\right)^{p-1} u_{e}^{T} k_{e} u_{e}$.

The discrete parameter corresponds to $(p-1)$. By default, the discrete parameter is 1 for shell elements and 2 for solid-state elements.

The tools of structural optimization include the variability of topology and size. In setting optimization problems, parameters can be volume, mass, moment of inertia, centre of gravity, speed, acceleration, stresses, displacements, forces, and external functions. These values can also be used as optimization criteria (for example, the goal is to reduce the mass of the part). The result of topological optimization is the distribution of material on a given geometry with a specific set of loads and fixations.

The optimization tools include variety of topologies and sizes. In setting optimization problems, parameters can be volume, mass, moment of inertia, centre of gravity, speed, acceleration, stresses, displacements, forces, and external functions. These values can also be used as optimization criteria (the purpose of this work is to reduce the mass of the part). The result of topological optimization is the distribution of material on a given geometry with a certain set of loads and anchors. From an engineering point of view, the most desirable goal is to get a solution in which the porous regions are largely suppressed and then also in the design stage to get a solution consisting only of solid and empty regions [3]. 
The mathematical model for calculations was chosen as follows in Fig.1.

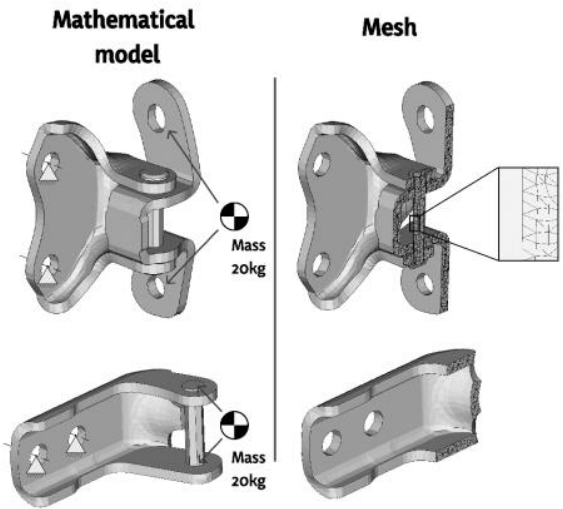

Fig. 1. The mathematical model and finite-element mesh of door hinge

The model is made from solid finite elements. The holes are directly attached to the car body are fitted with seals they are subject to the displacement condition. And on the holes that belong to the door mounting, there are concentrated masses, based on the calculation that the door weight is $200 \mathrm{~N}$.

The material properties of this FE model are defined as a linear-elastic material with a yield strength [4].

\section{RESULTS}

One of the most modern optimization methods in the field of applied mechanics is semi-automatic product development (generative design), which allows the engineer to get a readymade solution based on a single optimization calculation, rather than multiple iterations, as it was before. Automation of the process is achieved by machine learning, used in modern software, which allows you to get not just a concept, but a ready-to-manufacture part, in the shortest possible time, the production parameters of which are already taken into account in the calculations.

Software products that take into account the type of production (milling, 3D printing, casting, etc.) were used. The calculation process used machine learning, which is trained using special algorithms, parallel computing and cloud technologies to speed up the solution of tasks [5]-[12].

The most breakthrough technologies in the field of semiautomatic product development software are use of cloud technologies and parallel computing.

The cloud technologies are allowing to create a huge database of results of optimization calculations for the purpose of training the neural network. Another important feature is the ability not to use the initial geometry in the calculation, because only 2 types of areas are required to start optimization: areas that we want to leave in the future geometry (such as holes, mounting pads, etc.) and areas that should not contain any material (for example, the area inside the hole where the fastener should be located). This function is achieved using machine learning in cloud programs.
Parallel computing, for his part, can significantly reduce the speed of optimization calculations and visualization. Most of modern scientific software use power of NVIDIA GPUs and parallel computing CUDA, so we decided to use it in our research.

In GPU-accelerated applications, the sequential part of the workload runs on the CPU - which is optimized for singlethreaded performance - while the compute intensive portion of the application runs on thousands of GPU cores in parallel. When using CUDA, developers program in popular languages such as $\mathrm{C}, \mathrm{C}++$, Fortran, Python and MATLAB and express parallelism through extensions in the form of a few basic keywords [13]-[15].

In this paper, CUDA uses the resources of the computer's video card to speed up the optimization process and interpret its results by circumscribing the neural network, since we have a finite element grid at the exit from the optimization process, and interpreting the grid using surfaces is quite resourceintensive.

The block-diagram of generative design method using cloud-based technologies and parallel computing using CUDA are provided (Fig.2-3).

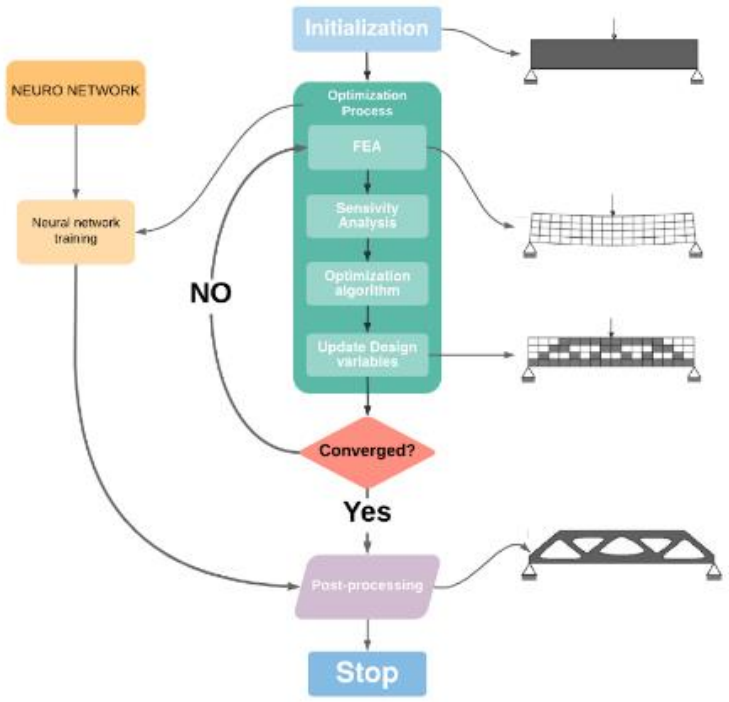

Fig. 2. Block diagram of the generative design process

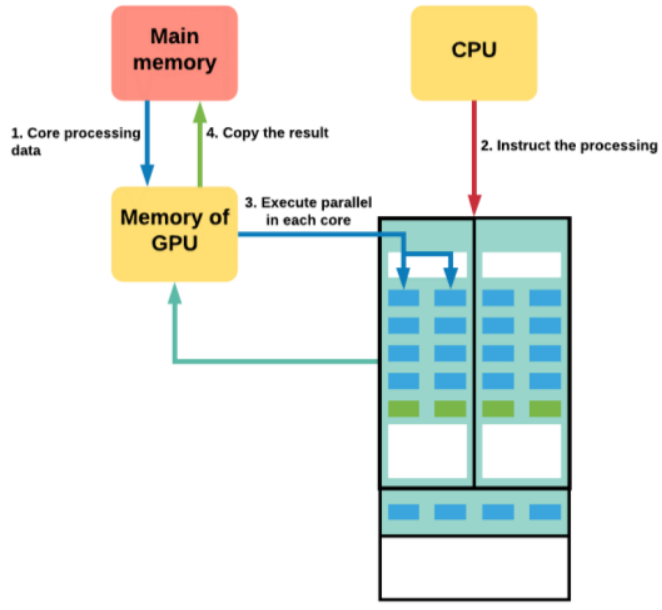

Fig. 3. Block diagram of the CUDA parallel computing 
Using generative design technology, an engineer can get a ready-to-manufacture model that may have some shape defects if the mathematical model is set incorrectly during postprocessing. After setting the task, we launched an optimization analysis. Figures 4-5 shows the result of optimization car door hinges.
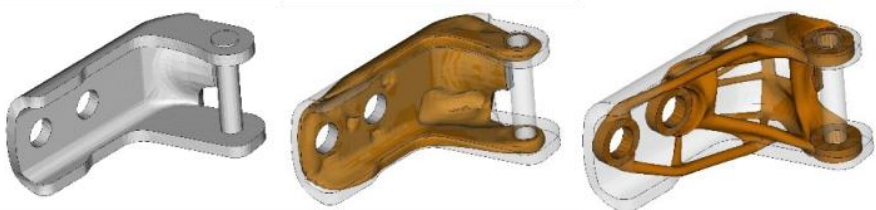

Fig. 6. Result of generative design optimization of upper hinges of a door (left - starting shape, central - 2nd step, right - final result)
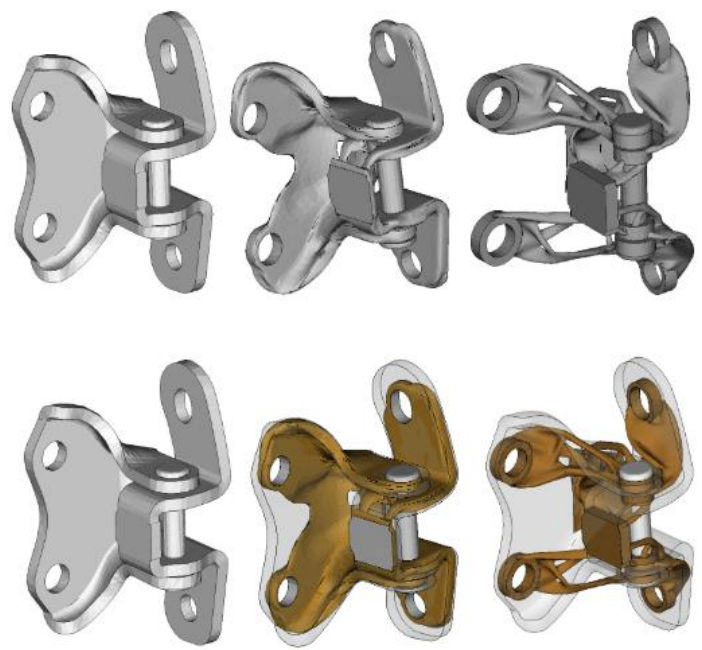

Fig.5. Result of generative design optimization of lower hinges of a door (left - starting shape, central - 2nd step, right - final result)

During the optimization, thanks to the algorithm described above, the neural network was trained to search for the optimal option based on similar calculations performed by us in the laboratory earlier. As a result, a solid-state model was obtained using the interpretation of the finite element grid obtained in the program.

After optimization the data of the volume change in each iteration was uploaded and graphs of the volume dependence on the optimization iteration were constructed (Fig.6).

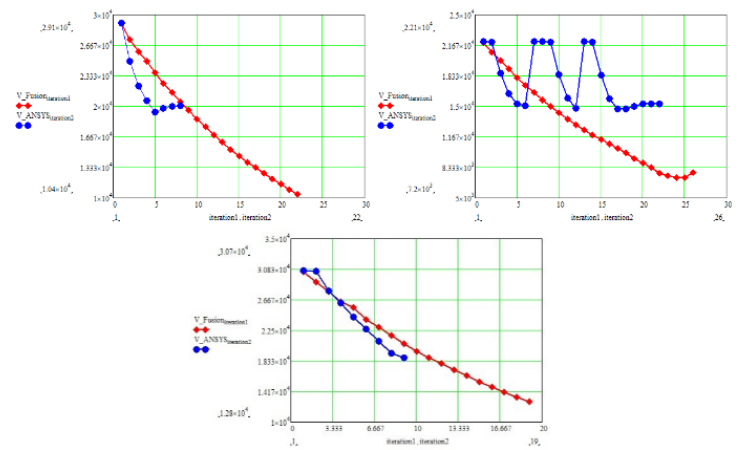

Fig.6. Graph of volume reduction depending on the optimization number (Red - cloud-based software, Blue - Parallel computing)
From Fig. 6, you can see that the use of a neuro network technologies has a noticeable advantage in optimization speed compared to classical optimizers based on parallel computing.

After optimization, verification static calculations were performed, which resulted in stress and strain fields for both the initial geometry and the two optimized variants.

The strain and stress fields of the initial geometry are shown in (Fig.7-8).
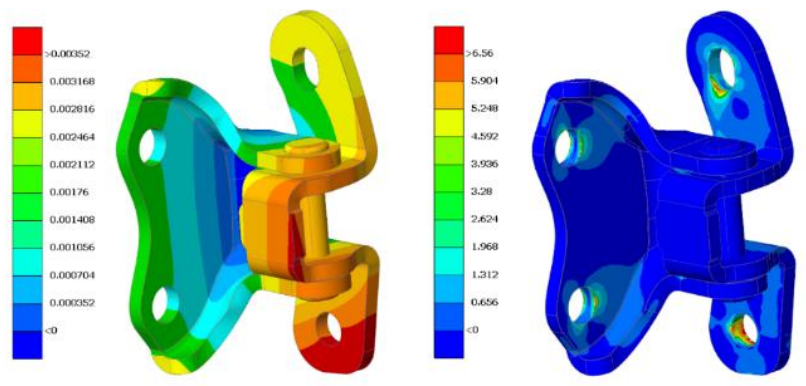

Fig.7. Result of static analysis of lower hinges of a door starting shape (Left-deformation, Right-Stress)
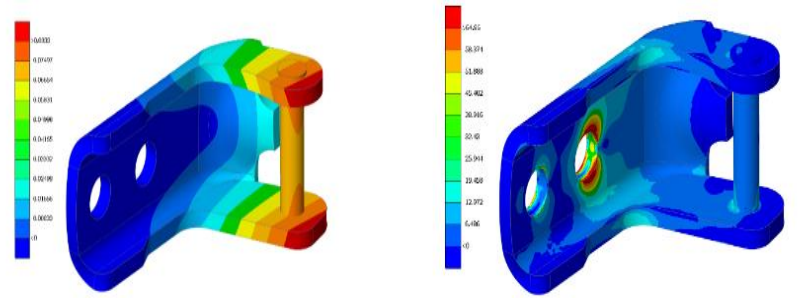

Fig.8. Result of static analysis of upper hinges of a door starting shape (Left-deformation, Right-Stress)

As we can see, the results of the verification calculation of the geometry obtained with parallel computing tools (Fig.9-10) differ slightly from the results of the initial geometry. The upper hinge has even better indicators than the base one.
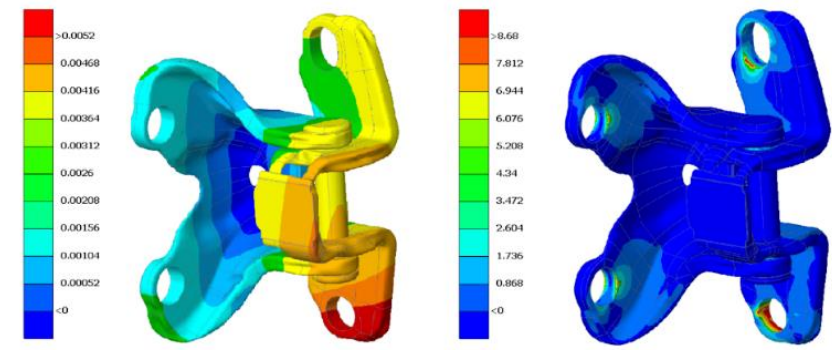

Fig.9. Result of static analysis of lower hinges of a door optimized with parallel computing (Left-deformation, Right-Stress)
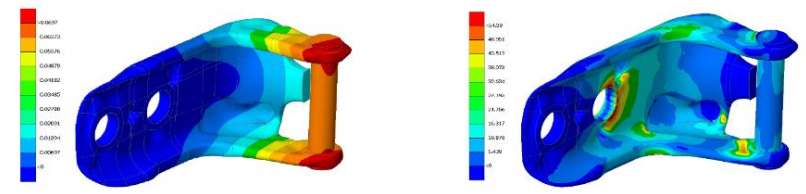

Fig.10. Result of static analysis of upper hinges of a door optimized with parallel computing (Left-deformation, Right-Stress) 
The results of the geometry obtained by optimized with cloud-based technologies (Fig.11-12), despite good indicators of mass reduction and accurate interpretation of the geometry, are an order of magnitude lower than the results of deformation and stresses of the base geometry.

There is a stress concentrator point on the upper hinge. Based on the stress values at this point, we can conclude that it poses a greater threat of failure (Fig.13).

This problem can be solved by "manually fine-tune" this part by increasing the thickness of the stiffeners.
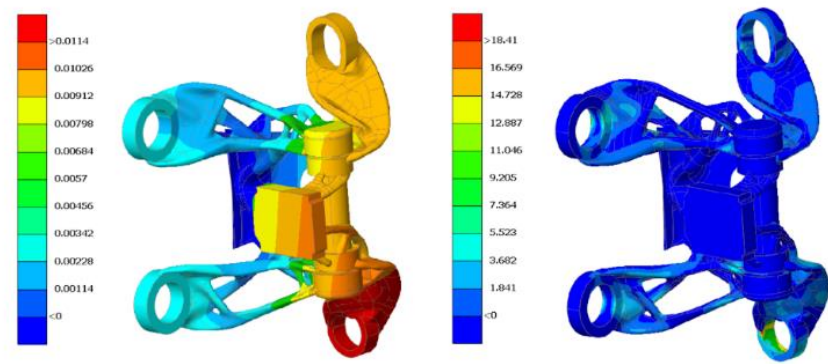

Fig.11. Result of static analysis of lower hinges of a door optimized with cloud-based technologies (Left-deformation, Right-Stress)
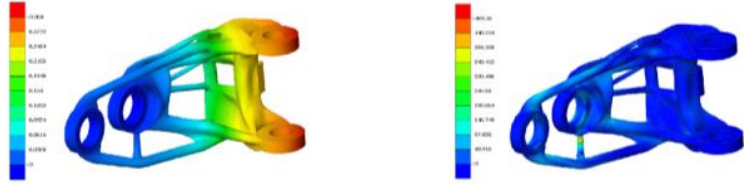

Fig.12. Result of static analysis of upper hinges of a door optimized with cloud-based technologies (Left-deformation, Right-Stress)

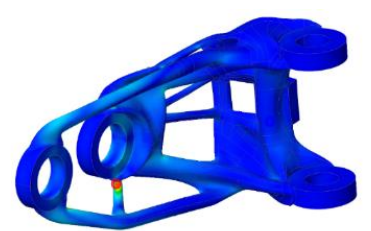

Fig.13. Result of static analysis of upper hinges of a door optimized with cloud-based technologies (Left-deformation, Right-Stress)

Similar challenge in this area was carried out by General motors. In this article the company not only shows the optimized car seat bracket but also gives information about its strategy to support and implement the generative design and additive manufacturing in company [2].

Generative design helps solving challenges not only in automotive industry but also in wide range of engineering cases. As an example, generative design is quite popular in architecture. Generative design was used by researchers in Lisboan technical University to design a Market building. As the researches claims it becomes possible to save a significant amount of time and effort by defining a library containing generalized implementations of the corresponding algorithms [16].

\section{RESULTS AND DISCUSSION}

The results of strength calculations showed that the software that used the generative design approach facilitates the construction of door hinges by a greater percentage, but at the same time loses to ANSYS according to the results of the verification calculation - it has large deformations and stresses. This means that the mathematical model for software with generative design requires further work and comparative calculations with other similar solutions on the market.

\section{CONCLUSION}

Optimization of vehicle door hinges with maintaining mechanical characteristics was the main goal of our research. We also wanted to show the most modern approaches for calculating and optimizing parts based on generative design. The generative design itself has an advantage over conventional topological optimization in the accuracy of the result and automatic interpretation of the analysis results. In addition, the time that takes up the optimization process, both when using cloud-based technologies and parallel computing using GPU.

We presented the original geometric model of electric car door hinges together with the block diagram of the optimizer. For $N=22$, where $N$ is the average number of calculated iterations with cloud-based software, and $N_{2}=13$ is the average number of calculated iterations with parallel computing.

During optimization, we were able to reduce the volume by $30 \%$ and create a part that meets the criteria for mechanical characteristics. To confirm the positive result of the calculation, we used an optimized geometry and performed static structural calculations. For this part, the type of manufacturing was selected - die casting as its cost in the automotive industry is much cheaper than additive manufacturing or milling.

As mentioned earlier, software with a generative design loses to ANSYS based on the results of the verification calculation, so the use of ANSYS in this task is optimal because it allows you to preserve the mechanical characteristics while reducing the material used for production.

A prototype was made, and experimental studies were conducted. The prototype is a modified CAD model of the original design. The properties of the prototype and the developed optimized hinges are compared.

\section{References}

[1] G. Rozvany, M. Zhou and T. Birker, "Generalized shape optimization without homogenization", Structural Optimization, vol. 4, no. 3-4, pp. 250-252, 1992.

[2] C. Razvan, "Overview of structural topology optimization methods for plane and solid structures", annals of the oradea university. Fascicle of Management and Technological Engineering., vol., 20143, no. 3, 2014. 
[3] V. Goryunov, "Topological optimization of the protective curtain node of the ITER optical diagnostic module", Master's thesis, pp. 16-17, 2017.

[4] V. Horová, M. Bojko and J. Dobeš, "Methodology of using the Adjoint solver optimization tool during flow in the intercooler filling line to minimize pressure drop", EPJ Web of Conferences, vol. 213, p. 02025, 2019.

[5] ANSYS, Theory Guide, ANSYS, Inc., 2019.

[6] R. Rao and G. Waghmare, "A new optimization algorithm for solving complex constrained design optimization problems", Engineering Optimization, vol. 49, no. 1, pp. 60-83, 2016.

[7] J. Martínez-Frutos, P. Martínez-Castejón and D. HerreroPérez, "Efficient topology optimization using GPU computing with multilevel granularity", Advances in Engineering Software, vol. 106, pp. 47-62, 2017.

[8] J. Martínez-Frutos and D. Herrero-Pérez, "GPU acceleration for evolutionary topology optimization of continuum structures using isosurfaces", Computers \& Structures, vol. 182, pp. 119-136, 2017.

[9] A. Mirzendehdel, M. Behandish and S. Nelaturi, "Topology optimization with accessibility constraint for multi-axis machining", Computer-Aided Design, vol. 122, p. $102825,2020$.

[10] J. Martínez-Frutos and D. Herrero-Pérez, "Large-scale robust topology optimization using multi-GPU systems", Computer Methods in Applied Mechanics and Engineering, vol. 311, pp. 393-414, 2016.

[11] S. Zheng, W. Tang and B. Li, "A new topology optimization framework for stiffness design of beam structures based on the transformable triangular mesh algorithm", Thin-Walled Structures, vol. 154, p. 106831, 2020.

[12] L. Mussi, F. Daolio and S. Cagnoni, "Evaluation of parallel particle swarm optimization algorithms within the CUDA $^{\mathrm{TM}}$ architecture", Information Sciences, vol. 181, no. 20, pp. 4642-4657, 2011.

[13] NVIDIA, CUDA Theory Guide, NVIDIA Corporation.

[14]Dr. Brian Tuominen, "Hands-on Programming with CUDA and Python", Packt Publishing, 2018.

[15] N. Maclaren, "Why (and Why Not) to Use Fortran Instead of C++, Matlab, Python etc.", University of Cambridge Computing Service, 2012

[16]R. Fernandes, "Generative Design: a new stage in the design process", Master's thesis, pp. 45-98, 2013.
Creative Commons Attribution License 4.0 (Attribution 4.0 International, CC BY 4.0)

This article is published under the terms of the Creative Commons Attribution License 4.0

https://creativecommons.org/licenses/by/4.0/deed.en_US 